\title{
Avaliação da satisfação e resolubilidade da plataforma telessaúde redes no estado do Pará, Brasil
}

\author{
Assessment of satisfaction and resolvability of the Telehealth Network Platform in the state of \\ Pará, Brazil
}

\section{Evaluación de satisfacción y resolubilidad de la plataforma telesalud en el estado de Pará, Brasil}

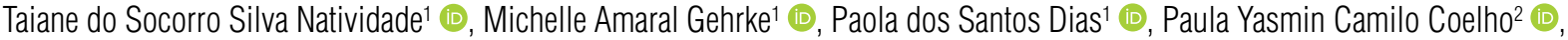 \\ Patrícia Oliveira Bezerra2 ${ }^{(10}$, Ana Carla Carvalho de Magalhães ${ }^{1}$ (1), Napoleão Braun Guimarães ${ }^{1}$ (1)
}

1Universidade do Estado do Pará - Belém (PA), Brasil.

${ }^{2}$ Centro Universitário Metropolitano da Amazônia - Belém (PA), Brasil.

\section{Resumo}

Introdução: As tecnologias da informação modernizaram diversos ramos de atuação humana, inclusive a medicina. Nesse contexto, surge a telemedicina como um avanço das tecnologias de comunicação aplicadas nessa área. No Brasil, o Telessaúde é uma ferramenta da telemedicina a serviço da saúde pública e funciona com o propósito de elevar a resolubilidade da Atenção Primária à Saúde (APS). No Pará, Norte do Brasil, o núcleo Telessaúde presta assistência aos 144 municípios do estado. Objetivo: Avaliar a resolubilidade dos casos enviados por teleconsultorias e a satisfação dos profissionais solicitantes no Telessaúde Redes Núcleo Pará, de 2015 a 2019. Métodos: Foi realizado um estudo observacional, transversal e quantitativo de série histórica, no qual se consultou a plataforma Telessaúde para verificação dos dados sobre resolubilidade, satisfação profissional e status de utilização do serviço, com base em perguntas geradas automaticamente na plataforma. Resultados: Verificou-se que a resolubilidade variou ao longo dos anos com 45,6 a $70 \%$ de referenciamentos evitados, demonstrando-se relativamente pouco variável. Já a satisfação dos profissionais usuários demonstrou-se elevada, variando de 77,9 a $95,45 \%$, estando os solicitantes majoritariamente "satisfeitos" ou "muito satisfeitos" com o serviço. Conclusões: Os dados sugerem a relevância do programa no que tange ao apoio à APS, com efeitos diversos na saúde pública.

Palavras-chave: Telemedicina. Telemonitoramento. Atenção primária à saúde.

Como citar: Natividade TSS, Gehrke MA, Dias PS, Coelho PYC, Bezerra P0, Magalhães ACC, Guimarães NB. Avaliação da satisfação e resolubilidade da plataforma telessaúde redes no estado do Pará, Brasil. Rev Bras Med Fam Comunidade. 2021;16(43):2411. https://doi.org/10.5712/rbmfc16(43)2411

\author{
Autor correspondente: \\ Taiane do Socorro Silva Natividade \\ E-mail: ts.natividade@gmail.com \\ Fonte de financiamento: \\ não se aplica. \\ Parecer CEP: \\ 2.951.049. \\ Procedência: \\ não encomendado. \\ Avaliação por pares: \\ externa. \\ Recebido em: 24/02/2020. \\ Aprovado em: 05/08/2021. \\ Editor Associado: \\ Leandro David Wenceslau
}




\begin{abstract}
Introduction: Information technologies have modernized several branches of human activity, including medicine. In this context, telemedicine emerges as an advance in communication technologies applied to medicine. In Brazil, Telehealth is a telemedicine tool in service of public health, and its purpose is to improve the resolvability of Primary Health Care. In the state of Pará, north of Brazil, the Telehealth platform provides assistance to the 144 municipalities in the state. Objective: To assess the resolvability of cases forwarded by teleconsultation and the satisfaction of requesting professionals with the Pará Telehealth Network Center, from the years of 2015 to 2019. Methods: An observational, cross-sectional and quantitative study of a historical series was carried out, in which the Telehealth Platform was consulted to verify data on resolvability, professionals' satisfaction, and service usage status based on questions automatically generated on the platform. Results: Over the years, resolvability ranged between $45.6 \%$ and $70 \%$ of avoided referrals to other services, proving to be slightly variable. The satisfaction of professionals/platform users was high, ranging from $77.9 \%$ to $95.45 \%$, with most requesters being "satisfied" or "very satisfied" with the service. Conclusions: The data suggest the relevance of the program in terms of Primary Health Care support, producing different effects on public health.
\end{abstract}

Keywords: Telemedicine. Telemonitoring. Primary health care.

\title{
Resumen
}

Introducción: Las tecnologías de la información han modernizado varias ramas de la actividad humana, incluida la medicina. En este contexto, la telemedicina surge como un avance en las tecnologías de comunicación aplicadas en medicina. En Brasil, Telehealth es una herramienta de telemedicina al servicio de la salud pública, y trabaja con el propósito de aumentar la resolución de la Atención Primaria de Salud (APS). En Pará, norte de Brasil, el centro de Telesalud brinda asistencia a los 144 municipios del estado. Objetivo: Evaluar la resolución de casos enviados por teleconsulta y la satisfacción de los profesionales solicitantes en Telehealth Redes Núcleo Pará, de 2015 a 2019. Métodos: Para esto, se realizó un estudio observacional, transversal y cuantitativo de una serie histórica, que consultó a la plataforma de telesalud para verificar los datos sobre resolubilidad, satisfacción laboral y estado de uso del servicio, en base a preguntas generadas automáticamente en la plataforma. Resultados: Se encontró que la resolubilidad varió a lo largo de los años entre el $45,6 \%$ y el $70 \%$ de las derivaciones evitadas, demostrando ser relativamente poco variable a lo largo de los años, ya que la satisfacción de los usuarios profesionales fue alta, variando de 77,9\% a 95,45\%, y la mayoría de los solicitantes están "satisfechos" o "muy satisfechos" con el servicio. Conclusiones: Los datos sugiere la relevancia del programa en términos de apoyo de APS y con diferentes efectos sobre la salud pública.

Palavras-clave: Telemedicina. Telemonitorización. Atención primaria de salud.

\section{INTRODUÇÃO}

A conferência de Alma Ata transformou os sistemas de saúde de todo o mundo ao preconizar os cuidados primários em saúde como a porta de entrada da assistência em saúde. ${ }^{1}$ No Brasil, a VIII Conferência Nacional de Saúde ${ }^{2}$ ocorrida em 1986 consolidou a modalidade de acesso à saúde por meio da Atenção Primária à Saúde (APS), na Constituição Brasileira, e da Lei 8080/90, com o formato do Sistema Único de Saúde (SUS).

O desenvolvimento tecnológico contribuiu grandemente para os avanços em saúde, não só no que diz respeito à produção de novos medicamentos e possibilidades de tratamento, mas também quanto à disseminação das informações e seus impactos no processo saúde/doença. ${ }^{3}$

Nesse contexto, a telemedicina surge como ferramenta assistencial e de educação continuada aos profissionais de saúde. ${ }^{4,5}$ No Brasil, o Telessaúde é uma das ferramentas da telemedicina, é utilizado no meio de saúde coletiva e conta com uma plataforma on-line que não tem fins lucrativos para grandes empresas, visto que se trata de uma iniciativa do Governo Federal para a melhoria da saúde pública no Estado. Portanto, o Telessaúde é uma ferramenta de tecnologia assistiva que dá apoio a atividades em saúde com o uso da telemedicina, e que foi regulamentado por diversas leis até a criação do Programa Nacional Telessaúde Brasil Redes. ${ }^{6-8}$

Seu principal objetivo é ampliar a resolubilidade do SUS e diminuir a desigualdade nos acessos à saúde, aumentando a agilidade e reduzindo os custos de deslocamento de pacientes no nível da APS. Para 
isso, realiza suas atividades de teleconsultorias, tele-educação e telediagnóstico por meio da Plataforma Telessaúde. Todas essas modalidades de atendimento são consideradas instrumentos para oferecer segunda opinião formativa sobre casos de maior complexidade, que usualmente requerem o cuidado de um médico especialista. ${ }^{9,10}$ Dessa forma, a aliança entre medicina e tecnologia da comunicação promovida pelo Telessaúde é considerada um artefato de apoio a intervenções de condutas medicamentosas e não medicamentosas e a dúvidas acerca de sinais clínicos e exames complementares, de forma a permitir o acesso a diversos serviços de diferentes especialidades. ${ }^{11}$

Apesar de a telemedicina dividir opiniões no Brasil quanto a sua utilização, é relevante pesquisar o impacto da Plataforma Telessaúde na saúde pública brasileira, especialmente no contexto amazônico, em que se podem observar preocupantes indicadores de desenvolvimento e de saúde, além do número diminuto de profissionais atuantes na assistência. Isso pode acarretar dificuldade no acesso a serviços de saúde, frisando-se os atendimentos especializados. As dimensões geográficas da região, em parte, justificam o difícil acesso aos programas de saúde e, com isso, a utilização do Telessaúde pode se tornar uma possibilidade de aprimoramento profissional ou de apoio aos profissionais atuantes, ampliando o acesso da população à saúde, o que pode impactar a resolubilidade do sistema básico de saúde. ${ }^{12}$

Dessa forma, o objetivo deste artigo é analisar a resolubilidade alcançada pelas consultorias solicitadas, a satisfação dos profissionais solicitantes e o status de utilização da Plataforma Telessaúde quanto às repostas dos casos solicitados na plataforma.

\section{MÉTODOS}

\section{Desenho de estudo}

Trata-se de um estudo observacional, transversal e quantitativo de série histórica que se utilizou das informações da base de dados do Telessaúde Pará.

\section{Cenário de estudo}

Esta pesquisa foi realizada na sede do Núcleo de Telessaúde do Pará, situado na Universidade do Estado do Pará, campus Centro Ciências Biológicas e da Saúde, durante o mês de fevereiro de 2020 e utilizou as informações referentes ao período de $1^{\circ}$ de janeiro de 2015 a 31 de dezembro de 2019 para as teleconsultorias realizadas no estado do Pará. Neste trabalho, analisaram-se os dados coletados por meio da Plataforma Telessaúde Rio Grande do Sul, visto que essa é a plataforma utilizada pelo Núcleo Telessaúde Pará.

\section{Critérios de inclusão e exclusão}

Foram analisadas todas as teleconsultorias solicitadas dos anos de 2015 a 2019, reservando-se para análise aquelas que apresentavam dados sobre a resolubilidade e a satisfação profissional nos anos estabelecidos para a pesquisa. Sendo assim, foram excluídas as teleconsultorias em que não se preencheram os dados pesquisados, as que não foram realizadas no período indicado e as que foram canceladas pelos reguladores do sistema ou pelos profissionais solicitantes. 


\section{Metodologia proposta}

Os dados referentes à resolubilidade neste estudo dizem respeito especificamente ao não encaminhamento do paciente da atenção primária a outro nível de complexidade, portanto evitando referenciamentos aos níveis de complexidade superiores. Dessa forma, considerou-se que a teleconsultoria foi resolutiva ao finalizar o atendimento ainda na APS, sem realizar o encaminhamento.

Os dados foram coletados por meio da pergunta automática apresentada no sistema da plataforma Telessaúde após a resposta do teleconsultor, "a teleconsultoria evitou encaminhamento?", possível de ser respondida por meio das opções automáticas "sim" ou "não". Quanto à satisfação, após a leitura da reposta redigida pelo médico especialista sobre o relato de caso, a plataforma questiona "você está satisfeito com a resposta da teleconsultoria?" e oferece as opções "muito satisfeito", "satisfeito", "indiferente", "insatisfeito" e "muito insatisfeito" aos profissionais da saúde que solicitaram apoio ao Núcleo Pará.

\section{Análise de dados}

A análise foi realizada com dados brutos obtidos por meio de tabelas geradas automaticamente pela plataforma Telessaúde. Esses dados são provenientes dos atendimentos à população em todo o estado do Pará, uma vez que se busca avaliar a relevância do programa em todo o estado. Realizou-se análise descritiva de dados, expressos por números brutos, porcentagem e medidas de dispersão.

\section{Aspectos éticos}

Esta pesquisa obedeceu às normas da Resolução no 466/12 do Conselho Nacional de Saúde, que trata de pesquisas que envolvam seres humanos. Ao dispensar a assinatura do Termo de Consentimento Livre e Esclarecido, utilizou-se o Termo de Compromisso de Uso de Dados para a coleta.

O projeto foi submetido ao Comitê de Ética em Pesquisa situado na Universidade do Estado do Pará e aprovado segundo o parecer de número 2.951.049.

\section{RESULTADOS}

Nos anos de 2015 a 2019, as assistências do Telessaúde Pará alcançaram 1.969 teleconsultorias. Entretanto, somente 926 apresentavam dados sobre a quantidade de referenciamentos indicados em todo o estado, enquanto 1.232 traziam dados sobre a satisfação do profissional solicitante em relação à resposta do teleconsultor.

Essa discrepância entre os dados dá-se principalmente pelo fato de que não é obrigatório o preenchimento desses dois dados na plataforma Telessaúde. Assim, de acordo com os critérios de exclusão, o universo do estudo foi o total de 1.232 teleconsultorias avaliadas apenas para dados sobre satisfação e 926 avaliadas para os dados de referenciamento.

Neste estudo, a resolubilidade caracteriza-se pela capacidade de evitar referenciamento a um nível superior de atenção e complexidade, pois o serviço é apto a enfrentar e resolver a condição de saúde ainda na APS. A Tabela 1 apresenta os dados de resolubilidade, apontando a quantidade absoluta e a porcentagem de encaminhamentos a outros níveis de complexidade realizados ou evitados após a resposta do teleconsultor, de acordo com o ano. Observa-se discreto aumento dos encaminhamentos ao longo dos quatro anos avaliados. 
Tabela 1. Quantidades absolutas e porcentagens de encaminhamentos evitados e realizados após a resposta das teleconsultorias analisadas pelo Telessaúde Redes Pará nos anos de 2015 a 2019.

\begin{tabular}{lcccccccccc}
\hline \multirow{2}{*}{ Referenciamento } & \multicolumn{2}{c}{$\mathbf{2 0 1 5}$} & \multicolumn{2}{c}{$\mathbf{2 0 1 6}$} & \multicolumn{2}{c}{$\mathbf{2 0 1 7}$} & \multicolumn{2}{c}{$\mathbf{2 0 1 8}$} & \multicolumn{2}{c}{$\mathbf{2 0 1 9}$} \\
\cline { 2 - 13 } & $\mathbf{n}$ & $\%$ & $\mathbf{n}$ & $\%$ & $\mathbf{n}$ & $\%$ & $\mathbf{n}$ & $\%$ & $\mathbf{n}$ & $\%$ \\
\hline Evitados & 35 & 70 & 154 & 59,9 & 144 & 29,9 & 89 & 65,44 & 21 & 45,6 \\
Realizados & 15 & 30 & 103 & 40,1 & 339 & 70,1 & 47 & 34,56 & 25 & 54,4 \\
N/A & 53 & - & 675 & - & 188 & - & 56 & - & 25 & - \\
Total & 103 & 100 & 932 & 100 & 671 & 100 & 192 & 100 & 71 & 100 \\
\hline
\end{tabular}

Fonte: Telessaúde Redes Pará; 2020. n: número de teleconsultorias; \%: porcentagem; N/A: não se aplica.

No que diz respeito à satisfação do profissional solicitante quanto às repostas obtidas por meio das teleconsultorias, a Tabela 2 estratifica as respostas em "muito satisfeitos", "satisfeitos", "indiferente" "insatisfeitos" e "muito insatisfeitos". Observam-se ao longo dos anos taxas semelhantes quanto à satisfação, exceto em 2019, quando houve acentuada queda.

Tabela 2. Satisfação profissional em quantidade absoluta e porcentagem quanto às respostas das teleconsultorias realizadas no Telessaúde Redes Pará nos anos de 2015 a 2019.

\begin{tabular}{lcccccccccc}
\hline \multirow{2}{*}{ Nível de satisfação } & \multicolumn{2}{c}{$\mathbf{2 0 1 5}$} & \multicolumn{2}{c}{$\mathbf{2 0 1 6}$} & \multicolumn{2}{c}{$\mathbf{2 0 1 7}$} & \multicolumn{2}{c}{$\mathbf{2 0 1 8}$} & \multicolumn{2}{c}{$\mathbf{2 0 1 9}$} \\
\cline { 2 - 12 } & $\mathbf{n}$ & $\%$ & $\mathbf{n}$ & $\mathbf{0}$ & $\mathbf{n}$ & $\%$ & $\mathbf{n}$ & $\%$ & $\mathbf{n}$ & $\%$ \\
\hline Muito insatisfeito & 0 & 0 & 11 & 1,9 & 8 & 1,7 & 2 & 1,8 & 2 & 5,5 \\
Insatisfeito & 1 & 2,32 & 12 & 2,1 & 16 & 3,45 & 3 & 2,75 & 2 & 5,5 \\
Indiferente & 1 & 2,32 & 9 & 1,5 & 16 & 3,45 & 0 & 0 & 4 & 11,1 \\
Satisfeito & 14 & 32,56 & 252 & 43,6 & 235 & 50,4 & 43 & 39,45 & 12 & 33,4 \\
Muito satisfeito & 27 & 62,8 & 294 & 50,9 & 191 & 41 & 61 & 56 & 16 & 44,5 \\
N/A & 60 & - & 354 & - & 205 & - & 83 & - & 35 & - \\
Total & 103 & 100 & 932 & 100 & 671 & 100 & 192 & 100 & 71 & 100 \\
\hline
\end{tabular}

Fonte: Telessaúde Redes Pará; 2020. n: número de teleconsultorias; \%: porcentagem; N/A: não se aplica.

A utilização da Plataforma Telessaúde ao longo dos anos é indicada na Tabela 3. Demonstra-se ao longo dos anos o número de Unidades Básica de Saúde (UBS) que solicitaram teleconsultorias na Plataforma Telessaúde. Foram estratificadas ao longo dos anos as UBS que apresentavam: menos de 12 teleconsultorias por ano, número considerado abaixo do padrão; entre 12 e 36 teleconsultorias por ano, número considerado a média; e acima de 36 teleconsultorias por ano.

Tabela 3. Utilização da Plataforma Telessaúde, de acordo com os anos pesquisados, indicando a média de utilização por Unidade Básica de Saúde em número brutos.

\begin{tabular}{lccccc}
\hline Utilização & $\mathbf{2 0 1 5}$ & $\mathbf{2 0 1 6}$ & $\mathbf{2 0 1 7}$ & $\mathbf{2 0 1 8}$ & $\mathbf{2 0 1 9}$ \\
\hline Número de UBS com <12 teleconsultorias & 52 & 268 & 104 & 67 & 38 \\
Número de UBS com 12 a 36 teleconsultorias & 1 & 6 & 8 & 0 & 0 \\
Número de UBS com >36 teleconsultorias & 0 & 1 & 2 & 2 & 0 \\
Total & 53 & 275 & 114 & 68 & 38 \\
\hline
\end{tabular}

Fonte: Telessaúde Redes Pará; 2020. UBS: Unidade Básica de Saúde. 


\section{DISCUSSÃo}

\section{Resumo dos principais achados}

Neste estudo, avaliou-se a resolubilidade da assistência via teleconsultoria e a satisfação profissional com as respostas recebidas mediante a pergunta automática gerada nas teleconsultorias efetivadas no estado do Pará, qual seja: "a teleconsultoria evitou encaminhamento?" A questão podia ser respondida por meio das opções automáticas "sim" ou “não". Observou-se resolubilidade variável de 45,6 a 70\% entre os anos de 2015 a 2019, período em que se pôde observar queda nas porcentagens de resolubilidade nos anos de 2016, 2017 e 2019, acentuada no ano de 2017.

Após a leitura da reposta redigida pelo médico especialista sobre o relato de caso, a plataforma questiona ao profissional solicitante: "você está satisfeito com a resposta da teleconsultoria?", oferecendo as opções "muito satisfeito", "satisfeito", "indiferente", "insatisfeito" e "muito insatisfeito". Quanto à satisfação, verificaram-se médias elevadas ao longo dos anos de 2015 a 2018, porém com queda no ano de 2019, o que coincide com a reduzida resolubilidade e os menores índices de satisfação em 2019.

Verificou-se também a média de utilização de acordo com o número de solicitações realizadas na Plataforma Telessaúde e observou-se redução no número de UBS que solicitaram teleconsultorias, bem como a frequente utilização abaixo de 12 teleconsultorias por UBS.

\section{Comparação com a literatura já existente}

Segundo a Organizacion Panamericana de la Salud, ${ }^{13}$ a APS deve ser a porta de acesso para a atenção à saúde, logo precisa oferecer assistência longitudinal e integral à população. ${ }^{14} \mathrm{~A}$ fim de oferecer assistência à APS, o Programa Telessaúde Brasil Redes expande-se desde 2007 no país, apesar das discussões sobre sua formalização na classe médica. ${ }^{15}$ Neste trabalho foi analisada a resolubilidade do programa e a satisfação profissional dos usuários do estado do Pará.

A resolubilidade é definida pelo SUS como a capacidade do serviço de saúde para enfrentar e resolver um problema de acordo com o seu nível de competência, portanto evitando referenciamentos desnecessários a outros níveis de complexidade, o que neste trabalho é explicitado por meio da quantidade de encaminhamentos realizados após a resposta da teleconsultoria.

Entretanto, ressalta-se que o referenciamento a um novo nível de complexidade não necessariamente significa que a assistência da teleconsultoria na APS não tenha sido resolutiva, visto que os dados brutos não permitem identificar se o encaminhamento foi desnecessário. Por conseguinte, infere-se que por meio da teleconsultoria foi possível verificar que o caso clínico em questão não poderia ser elucidado ainda na APS e, dessa forma, houve necessidade de acesso presencial a um especialista.

Já a satisfação é evidenciada exclusivamente por meio da satisfação do profissional solicitante quanto às informações recebidas da plataforma Telessaúde. Tanto a necessidade de referenciamento a outro nível de complexidade quanto a satisfação são avaliadas por perguntas automáticas geradas pela plataforma. ${ }^{16,17}$

O Telessaúde possibilita a oferta remota de assistência especializada à população, favorecendo maior possibilidade de troca e a aquisição de novos conhecimentos entre os profissionais solicitantes e os especialistas, situados na capital do estado. ${ }^{18}$ Primeiramente, a plataforma prevê que a consulta presencial seja realizada por meio de um profissional da saúde atuante na UBS e, em caso de dúvidas 
quanto ao diagnóstico ou necessidade de segunda opinião profissional, usualmente quando se mostra necessário atenção especializada, oferece profissionais especializados para auxiliar as equipes das UBS, em contato direto com a população por meio da teleconsultoria.

Para controle organizacional, o Ministério da Saúde estabeleceu como média de teleconsultorias por UBS de uma a três solicitações mensais, porém a demanda dos profissionais no Brasil pelo serviço do Telessaúde é considerada baixa. Essa situação intensifica-se no Pará, onde, apesar de todos os municípios estarem cadastrados no programa, quase metade das solicitações ao serviço provém de poucas cidades cadastradas. Neste trabalho é demonstrada a subutilização do serviço, corroborando estudos que constataram que clínicos preferem encaminhar pacientes a outros níveis de atenção e que, apesar do número considerável de cadastros na plataforma, o número de teleconsultorias enviadas é significativamente inferior. ${ }^{18,19}$

É válido ressaltar que diversos aspectos impactam a subutilização do serviço, entre eles a dificuldade de acesso à internet. ${ }^{8,20}$ De acordo com Fernandes, ${ }^{21}$ a conectividade no Brasil é um grande entrave para a atuação das consultorias a distância, pois diversas regiões brasileiras possuem internet com baixo desempenho e velocidade, empecilho ressaltado pelos profissionais de saúde. Na região Norte, embora haja melhorias, percebem-se limitações importantes na velocidade da conectividade e maior índice de uso urbano em relação ao rural. ${ }^{20}$ Entretanto, as novas tecnologias exigem um suporte tecnológico mínimo das áreas atendidas para que possam funcionar adequadamente.

Diversos núcleos de telessaúde no Brasil também têm demonstrado que a simples oferta de teleconsultoria não é o suficiente para aumentar a utilização do serviço pelos profissionais de saúde, segundo o estudo de Maeyama e Calvo, ${ }^{22}$ dada certa resistência profissional em sua utilização. $O$ trabalho também discorre sobre a possibilidade de tornar o uso do Telessaúde compulsório, a depender da gestão da UBS, pois a resolubilidade da APS permanece baixa. Isso pode se relacionar à falta de estrutura atual e também à realização de referências desnecessárias a níveis mais complexos, já que essas demandas poderiam ser elucidadas ainda na APS.

No caso de uso compulsório, a obrigatoriedade do fluxo de teleconsultorias teria lugar somente antes de o médico da UBS referenciar o paciente para um nível maior de complexidade, ou seja, a teleconsultoria poderia nortear a necessidade real do encaminhamento. Entretanto, ainda caberia ao médico da APS a decisão final, atuando a teleconsultoria unicamente como uma ferramenta de auxílio ao médico solicitante. ${ }^{22}$

A utilização reduzida da plataforma pode ser atribuída também ao discreto investimento público em telemedicina no SUS, tanto no que diz respeito à divulgação do serviço como no que tange ao esclarecimento profissional quanto à finalidade da plataforma. Esta muitas vezes necessita que sejam elucidados os benefícios de sua utilização na saúde pública, benefícios esses principalmente relacionados ao auxílio na educação continuada e ao impacto na resolubilidade ao se evitar o referenciamento desnecessário, gerando impacto positivo na economia dos cofres públicos e poupando verbas destinadas aos referenciamentos. ${ }^{23-25}$ Entretanto, ao se considerar que os atendimentos realizados em regiões remotas e sem estrutura limitam a atuação médica, o encaminhamento a outros níveis de complexidade torna-se inevitável, o que leva à diminuição da resolubilidade na APS. Esse fato é corroborado pelos resultados apresentados, nos quais se verifica a resolubilidade relativa das teleconsultorias solicitadas, como ocorrido no Núcleo Pará.

Ademais, existem dificuldades na manutenção de um serviço de qualidade que se expanda a outras regiões, uma vez que muitos profissionais não aderem ao Programa Telessaúde, bem como impasses acerca de licenciamento. Todavia, é válido frisar que houve aperfeiçoamento nessa área, com número crescente de serviços especialmente no exterior. ${ }^{26}$ 
Dada a complexidade envolvida no estabelecimento da telemedicina, cria-se o desafio de determinar como ela influencia na qualidade do serviço. ${ }^{26} \mathrm{~A}$ literatura descreve sua aplicação bem-sucedida e evita potenciais hospitalizações. ${ }^{27-30}$

Quanto à satisfação, apesar da literatura restrita, devemos considerá-la como um tema abordável por diversas faces, tais como satisfação com o sistema, com a resposta da teleconsultoria com base nas expectativas do solicitante, com a obtenção de auxílio em situações críticas, com a precisão do diagnóstico, entre outros. ${ }^{31}$ Neste estudo, o objetivo foi avaliar a satisfação quanto às respostas da teleconsultorias, buscando evidenciar se as solicitações dos médicos da atenção básica foram correspondidas por meio da avaliação da satisfação do profissional.

Ressalta-se que foram excluídas as teleconsultorias em cujos dados não constavam respostas sobre resolubilidade e satisfação. Utilizou-se apenas a parcela das teleconsultorias que apresentava os dados referentes ao refereciamento e à satisfação, e, neles, puderam-se verificar taxas semelhantes de satisfação ao longo dos anos, com usuários em geral muito satisfeitos ou satisfeitos com as respostas das teleconsultorias. Entretanto, esses dados não apresentam obrigatoriedade de resposta na plataforma, o que se pode considerar uma limitação importante do estudo, visto que as teleconsultorias não avaliadas não foram consideradas como parte da amostra da pesquisa.

Diversos estudos ${ }^{32-36}$ destacam a facilidade de disseminação da informação e da possibilidade de aprimoramento profissional com as teleconsultorias, ressaltando o seu valor ao favorecerem a educação permanente em saúde por meio de um processo dialético de aprendizagem e trazendo benefícios tanto para usuários como para os profissionais atuantes. Isso porque o ambiente digital aproxima os profissionais da saúde e favorece os pacientes, os quais serão beneficiados com o conhecimento e a prática clínica de outros profissionais, não importando a distancia. ${ }^{33}$

Dessa forma, o Telessaúde conecta serviços de saúde, centros de referência e universidades renomadas, o que possibilita a educação permanente dos profissionais e gestores, evitando viagens desnecessárias e oferecendo suporte à tomada de providências.

Outro ponto crucial para o funcionamento do Telessaúde é a insegurança profissional quanto aos dados dos usuários. Rezende et al. ${ }^{37}$ afirmam que o sistema de informações deve possuir mecanismos de acesso restrito e limitado para cada perfil de usuário, ponto que deve ser levado em consideração para a manutenção da privacidade e da confidencialidade das informações em trâmite no Telessaúde. Um sistema de segurança eficiente gera adequada restrição dos dados a pessoas autorizadas, ou mesmo só permite que se revelem informações privadas dos usuários e pacientes que tenham aceitado previamente que determinados dados sejam expostos a outros profissionais por meio da plataforma.

\section{Fortaleza e limitações do estudo}

Todavia, apesar do apresentado, o Telessaúde revela pontos frágeis em sua atuação, visto que os dados selecionados para a análise no estudo provêm de perguntas automáticas geradas pelo sistema e que não apresentam obrigatoriedade de resposta, o que reduz o número de teleconsultorias avaliadas.

Ademais, a utilização de dados brutos apenas é uma limitação importante, já que os dados brutos não indicam com segurança o motivo do referenciamento.

A acentuada queda da utilização do serviço ao longo dos anos também pode configurar importante fator limitante, dado este que coincide com a retirada dos médicos cubanos da assistência na APS. Pode- 
se pressupor que esse fato seja uma possível causa da redução das solicitações ao longo dos anos, que, entretanto, não explicaria completamente a redução da utilização.

Uma vez que o preenchimento de dados como necessidade de referenciamento e satisfação não é obrigatório, ressaltamos ainda essa situação como uma limitação importante, visto que foram desconsideradas as teleconsultorias em que não pudemos inferir qual o nível de satisfação do profissional solicitante.

Outro aspecto limitante dá-se pelos reduzidos investimento e difusão de informações sobre o tema da telemedicina na atualidade, o que dificulta a adesão e a possibilidade de troca de aprendizados entre profissionais. De acordo com Cunha, ${ }^{34}$ para que se tenha um processo de educação permanente eficiente, é necessário o constante treinamento dos profissionais envolvidos, além de renovação continuada do conteúdo disponibilizado. A Organização Pan-Americana da Saúde ${ }^{38}$ corrobora essa afirmação, apontando como desafio a sensibilização dos gestores sobre a importância e a necessidade de investimento na APS. ${ }^{39-42}$

Entretanto, apesar das limitações apresentadas quanto à adesão, foram coletados dados de cerca de 1.232 teleconsultorias ao longo de cinco anos de atendimentos do Telessaúde no estado do Pará.

\section{Implicações para a pesquisa na área e/ou prática dos profissionais}

Este artigo apresenta pela primeira vez dados sobre resolubilidade e satisfação profissional do Núcleo Telessaúde Pará e aponta para a necessidade de maior exploração de dados envolvendo mais estudos sobre telemedicina e saúde pública no contexto amazônico.

Outra relevante implicação consiste na geração de novos conhecimentos e pontos prioritários quanto à utilização de telemedicina, ressaltando a capacidade resolutiva da APS e a necessidade de maiores estudos sobre a importância do Telessaúde no contexto de melhorias em saúde coletiva.

\section{CONCLUSÕES}

A Plataforma Telessaúde Brasil Redes Pará surgiu com o objetivo de auxiliar os princípios de integralidade, equidade e universalidade do SUS. Em sua atuação, visa ampliar o acesso da população, diminuindo o tempo de deslocamento e as dificuldades de acesso dos usuários por meio de tecnologias de informação, principalmente ao evitar referenciamentos desnecessários, uma vez que muitos casos poderiam ser completamente abordados na APS, ao se minimizarem as distâncias geográficas.

O presente estudo sugere resolubilidade relativamente estável com discreta redução ao longo dos anos. De forma semelhante, o grau de satisfação dos profissionais usuários da plataforma foi alto, o que sugere ainda a importância do programa no que tange ao apoio ao profissional da atenção básica e à assistência também por meio da educação continuada. Entretanto, a subutilização do serviço também se mostrou uma realidade.

Há necessidade de maiores pesquisas na área para que se tenham dados mais aprofundados sobre o impacto do programa Telessaúde na saúde pública, de forma que se busque conhecer as necessidades e fragilidades do programa para proporcionar-Ihe melhorias.

\section{AGRADECIMENTOS}

Agradecimentos à Universidade do Estado do Pará. 


\title{
CONFLITO DE INTERESSES
}

\author{
Nada a declarar.
}

\section{CONTRIBUIÇÕES DOS AUTORES}

TSSN: Administração do projeto, Análise formal, Conceituação, Curadoria de dados, Escrita primeira redação, Escrita - revisão e edição, Investigação, Metodologia. MAG: Curadoria de dados, Escrita - primeira redação, Escrita - revisão e edição, Investigação. PSD: Curadoria de dados, Escrita - primeira redação, Investigação. PYCC: Curadoria de dados, Escrita - primeira redação, Investigação. POB: Curadoria de dados, Escrita - primeira redação, Investigação. ACCM: Administração do projeto, Supervisão. NBG: Administração do projeto, Análise formal, Supervisão.

\section{REFERÊNCIAS}

1. Organização Mundial de Saúde. Conferência Internacional sobre Cuidados Primários de Saúde. Declaração de Alma-Ata. Alma-Ata, URSS, 6-12 de setembro de 1978. Disponível em: https://web.archive.org/web/20090419121612/http://www. opas.org.br/coletiva/uploadArq/Alma-Ata.pdf

2. Brasil. Ministério da Saúde. $8^{a}$ Conferência Nacional de Saúde. Brasília: Centro de Documentação do Ministério da Saúde; 1986. Disponível em: http://www.ccs.saude.gov.br/cns/pdfs/8conferencia/8conf_nac_anais.pdf

3. Mayema MA, Jasper CH, Nilson LG, Dolny LL, Cutolo LRA. Health promotion as technology for social transformation. Revista Brasileira de Tecnologias Sociais 2015;2(2):129-43. https://doi.org/10.14210/rbts.v2.n2.p129-143

4. Whitten P, Holtz B. Provider utilization of telemedicine: the elephant in the room. Telemed J E Health 2008;14(9):995-7. https://doi.org/10.1089/tmj.2008.0126

5. Wootton R. Telemedicine support for the developing world. J Telemed Telecare 2008;14 (3):109-14. https://doi.org/10.1258/ jtt.2008.003001

6. Brasil. Ministério da Saúde. Secretaria de Gestão da Educação na Saúde. Departamento de Gestão da Educação na Saúde. Telessaúde. Brasília: Ministério da Saúde; 2007.

7. Brasil. Ministério da Saúde. Programa Telessaúde Brasil para apoio à Estratégia de Saúde da Família no Sistema Único de Saúde. Brasília: Ministério da Saúde; 2010.

8. Brasil. Ministério da Saúde. Secretaria de Gestão do Trabalho e da Educação na Saúde. Apresentação da produção de atividades dos Núcleos de Telessaúde. Brasília: Ministério da Saúde; 2015.

9. Forbes RC, Rybacki DB, Johnson TB, Hannah-Gillis A, Shaffer D, Hale DA. A cost comparison for telehealth utilization in the kidney transplant waitlist evaluation process. Transplantation 2018;102(2):279-83. https://doi.org/10.1097/ TP.0000000000001903

10. Fragidis LL, Chatzoglou PD. Implementation of a nationwide electronic health record (EHR). Int J Health Care Qual Assur 2018;31(2):116-30. https://doi.org/10.1108/IJHCQA-09-2016-0136

11. Hofmeyer J, Leider JP, Satorius J, Tanenbaum E, Basel D, Knudson A. Implementation of telemedicine consultation to assess unplanned transfers in rural long-term care facilities, 2012-2015: a pilot study. J Am Med Dir Assoc 2016;17(11):1006-10. https://doi.org/10.1016/j.jamda.2016.06.014

12. Brasil. Ministério da Saúde. Departamento de informática do Sistema Único de Saúde. Informações de saúde, epidemiológicas e morbidade: banco de dados. Brasília: Ministério da Saúde; 2017.

13. Organizacion Panamericana de la Salud. Informe Dawson sobre el futuro de los servicios médicos y afines, 1920 . Washington: Organizacion Panamericana de la Salud; 1964. Disponível em: https://iris.paho.org/bitstream/handle/10665.2/1133/42178. pdf?sequence=1\&isAllowed=y

14. Starfield B. Atenção primária: equilíbrio entre necessidades de saúde, serviços e tecnologia. Brasília: UNESCO, Ministério da Saúde; 2002. Disponível em: https://www.nescon.medicina.ufmg.br/biblioteca/imagem/0253.pdf

15. Brasil. Presidência da República. Casa Civil. Subchefia para Asuntos Jurídicos. Lei no 8.080 , de 19 de setembro de 1990. Dispõe sobre as condições para a promoção, proteção e recuperação da saúde, a organização e o funcionamento dos serviços correspondentes e dá outras providências. Disponível em: http://www.planalto.gov.br/ ccivil_03/leis/l8080.htm

16. Harzheim E, Gonçalves MR, Umpierre RN, Siqueira ACS, Katz N, Agostinho MR, et al. Telehealth in Rio Grande do Sul, Brazil: bridging the gaps. Telemed J E Health 2016;22(11):938-44. https://doi.org/10.1089/tmj.2015.0210

17. Godoy SCB, Guimarães EMP, Assis DSS. Avaliação da capacitação dos enfermeiros em unidades básicas de saúde por meio da telenfermagem. Esc Anna Nery 2014;18(1):148-55. http://doi.org/10.5935/1414-8145.20140022 
18. Castro Filho ED, Harzheim E, Schmitz CAA, Siqueira AC. Telessaúde para atenção primária. In: Gusso G, Lopes, JMC (orgs.). Tratado de medicina de família e comunidade: princípios, formação e prática. Porto Alegre: Artmed; 2012. p. 395-403.

19. Schmitz CAA. Telessaúde como suporte assistencial para a Atenção Primária à Saúde no Brasil [tese de doutorado]. Rio Grande do Sul: Faculdade de Medicina da Universidade Federal do Rio Grande do Sul; 2015.

20. Barbosa AF. Banda larga no Brasil: um estudo sobre a evolução do acesso e da qualidade das conexões à internet. São Paulo: Comitê Gestor da Internet no Brasil; 2018.

21. Fernandes MM. Desenvolvimento de modelo de gestão de tecnologias no Instituto Nacional de Câncer [dissertação de mestrado]. Rio de Janeiro: Fundação Oswaldo Cruz, Escola Nacional de Saúde Pública Sergio Arouca; 2009.

22. Maeyama MA, Calvo MCM. The Integration of telehealth in regulation centrals: the teleconsulting as a mediator between primary care and specialized care. Rev Bras Educ Med 2018;42(2):63-72. http://doi.org/10.1590/1981-52712015v42n2rb20170125

23. Mendes EV. A construção social da atenção da Atenção Primária à saúde. Brasília: Conselho Nacional de Secretários de Saúde; 2015.

24. Cifuentes C, Romero E, Godoy J. Design and implementation of a telemedicine primary-level hospital and low-cost system to reduce unnecessary patient transfers. Telemed J E Health 2017;23(6):521-6. https://doi.org/10.1089/tmj.2016.0180

25. Spaulding R, Belz N, DeLurgio S, Williams AR. Cost savings of telemedicine utilization for child psychiatry in a rural Kansas community. Telemed J E Health 2010;16(8):867-71. https://doi.org/10.1089/tmj.2010.0054

26. Department of Health \& Human Services. Telehealth services. Rural health fact sheet series. Centers for Medicare \& Medicaid Services; 2014. Disponível em: https://telepsychiatry.ecu.edu/wp-content/pv-uploads/sites/350/2020/09/telehealth-servicesfact-sheet.pdf

27. Kahn JM, Le TQ, Barnato AE, Hravnak M, Kuza CC, Pike F, et al. ICU telemedicine and critical care mortality: a national effectiveness study. Med Care 2016;54(3):319-25. https://doi.org/10.1097/MLR.0000000000000485

28. Ferreira IG, Godoi DF, Perugini ER, Lancini AB, Zonta R. Teledermatologia: uma interface entre a atenção primária e atenção especializada em Florianópolis. Rev Bras Med Fam Comunidade 2019;14(41):e2003. https://doi.org/10.5712/ rbmfc14(41)2003

29. Morley JE. Telemedicine: coming to nursing homes in the near future. J Am Med Dir Assoc 2016;17(1):1-3. https://doi. org/10.1016/j.jamda.2015.10.012

30. Daras LC, Wang JM, Ingber MJ, Ormond C, Breg NW, Khatutsky G, et al. What are nursing facilities doing to reduce potentially avoidable hospitalizations? J Am Med Dir Assoc 2017;18(5):442-44. https://doi.org/10.1016/j.jamda.2017.02.007

31. Oliveira GL, Cardoso CS, Ribeiro ALP, Caiaffa WT. Physician satisfaction with care to cardiovascular diseases in the municipalities of Minas Gerais: cardiosatis-TEAM scale. Rev Bras Epidemiol 2011;14(2):240-52. http://doi.org/10.1590/ S1415-790X2011000200006

32. Garcia-Barbero M. El valor educativo de la telemedicina. Educ Méd 2006;9(suppl 2):38-43.

33. Andrade CSGC. Agentes comunitários de saúde e os desafios da educação permanente: reflexões sobre a experiência do programa telessaúde Brasil: núcleo Rio de Janeiro. J Bras Tele 2012;1(2):51-2. https://doi.org/10.12957/jbrastele.2012.6408

34. Cunha IS. Educação permanente em saúde e planejamento estratégico situacional: o caso da Secretaria Estadual de Saúde do Piauí [dissertação de mestrado]. Rio de Janeiro: Fundação Oswaldo Cruz, Escola Nacional de Saúde Pública Sergio Arouca; 2009

35. Cunha NCH, Zilberman V, Sales MRM, Santucci LAC. A tecnologia audiovisual utilizada para informar e capacitar os trabalhadores da Secretaria Municipal da Saúde de São Paulo. São Paulo: Secretaria Municipal de Saúde; 2011.

36. Faria MGA, David HMSL. Telessaúde Brasil redes - núcleo Rio de Janeiro: a educação permanente no trabalho de enfermeiros da atenção básica. J Bras Tele 2012;1(1):23-4. https://doi.org/10.12957/jbrastele.2012.6402

37. Rezende EJC, Melo MCB, Tavares EC, Santos AF, Souza C. Ética e telessaúde: reflexões para uma prática segura. Rev Panam Salud Pública 2010;28(1):58-65.

38. Organização Pan-Americana da Saúde. Gestão do Conhecimento em Saúde no Brasil: avanços e perspectivas. Brasília: Organização Pan-Americana da Saúde; 2009. Disponível em: https://iris.paho.org/bitstream/ handle/10665.2/34953/9788579670039 por.pdf?sequence=1\&isAllowed=y

39. Pessoa CG, Sousa L, Ribeiro AL, Oliveira TB, Silva JLP, Alkmim MBM, et al. Description of factors related to the use of the teleconsultation system of a large telehealth service in Brazil - the Telehealth Network of Minas Gerais. Journal of the International Society for Telemedicine and EHealth 2016;4(e4):1-9. Disponível em: https://journals.ukzn.ac.za/index.php/ JISfTeH/article/view/133

40. Oliveira DG, Frias PG, Vanderlei LCM, Vidal SA, Novaes MA, Souza WV. Análise da implantação do Programa Telessaúde Brasil em Pernambuco, Brasil: estudo de casos. Cad Saúde Pública 2015;31(11):2379-89. https://doi.org/10.1590/0102$311 \times 00125914$

41. Okoroafor IJ, Chukwuneke FN, Ifebunandu N, Onyeka TC, Ekwueme CO, Agwuna KK. Telemedicine and biomedical care in Africa: prospects and challenges. Niger J Clin Pract 2017;20(1):1-5. https://doi.org/10.4103/1119-3077.180065

42. Santos AF, D’Agostinho M, Bouskela MS, Fernandéz A, Messina LA, Alves HJ. Uma visão panorâmica das ações de telessaúde na América Latina. Rev Panam Salud Pública 2014;35(5/6):465-70. 